Cahiers d'études italiennes

\title{
Jolles, il Decameron e le forme brevi
}

Jolles, le Décaméron et les formes brèves

Jolles, the Decameron and the Short Forms of Narration

\section{Renzo Bragantini}

\section{(2) OpenEdition}

\section{Journals}

Edizione digitale

URL: http://journals.openedition.org/cei/3136

DOI: $10.4000 /$ cei.3136

ISSN: 2260-779X

\section{Editore}

UGA Éditions/Université Grenoble Alpes

\section{Edizione cartacea}

Data di pubblicazione: 30 décembre 2016

Paginazione: 109-124

ISBN: 978-2-84310-337-7

ISSN: 1770-9571

Notizia bibliografica digitale

Renzo Bragantini, «Jolles, il Decameron e le forme brevi», Cahiers d'études italiennes [Online], 23 | 2016 online dal 23 janvier 2017, consultato il 26 mars 2021. URL: http://journals.openedition.org/cei/3136 DOl: https://doi.org/10.4000/cei.3136 


\title{
JOLLES, IL DECAMERON E LE FORME BREVI
}

\author{
Renzo Bragantini \\ Università di Roma - La Sapienza
}

Non t'ho forse fatto altra volta cenno d'un soggetto comico, la storia del giovane che se ne va per il mondo «ad apprendere la paura» ed è però tanto sciocco che non l'impara mai? Figurati il mio sgomento quando mi son reso conto che costui altri non era che il giovane Siegfried, che conquista il tesoro e risveglia Brünnhilde!

(Da una lettera di Wagner a Theodor Uhlig del Io maggio I85 $\mathrm{I}^{\mathrm{I}}$ )

Grazie all'intelligente passione di Silvia Contarini, il lettore italiano ha a disposizione, da oltre un decennio, una consistente porzione (la selezione, coerentemente, riguarda solo l'attività letteraria, non quella, altrettanto rilevante, dello storico e interprete delle arti figurative) del lavoro critico di quella tragica e incisiva figura che è stato André Jolles²; tragica per la sua adesione dalla prima ora al nazismo (si iscrive al partito l'i maggio del 1933, un mese dopo l'incendio del Reichstag il 27 febbraio, cui fa seguito il trionfo nazionalsocialista alle elezioni del 5 marzo), causa della rottura della sua amicizia con Huizinga, nonché della fine della corrispondenza con Jeltje, figlia del primo matrimonio (più in là, nel I944,

I. Wagner si riferisce a una delle più celebri fiabe dei Grimm, Märchen von einem, der auszog, das Fürchten zu lernen (Fiaba di uno che parti per apprendere la paura), la quarta della raccolta; di essa si conoscono innumerevoli versioni con poche e lievi varianti, anche italiane (cfr. almeno Straparola, Piacevoli notti, IV 5; Giovannin senza paura, inaugurale delle Fiabe italiane curate da Calvino). Dato il carattere imperscrutabile del grande musicista, che varia dalla franchezza più esibita e persino impudica all'improntitudine massima, nulla si può inferire in ordine alla sincerità del suo sgomento.

2. A. Jolles, I travestimenti della letteratura. Saggi critici e teorici (1897-1932), S. Contarini (a cura di), premessa di E. Raimondi, Milano, Bruno Mondadori, 2003. 
Hitler gli consegna personalmente la «Goethe-Medaille für Kunst und Wissenschaft»); tragica, ancora, per la morte del figlio Jolle, sottufficiale dell'esercito tedesco, durante l'eroica resistenza di Stalingrado all'assedio della Wehrmacht e delle forze dell'Asse, e infine, dopo la denazificazione, per la scomparsa (22 febbraio 1946), probabilmente in seguito a suicidio ${ }^{3}$.

Non davvero per porre in prima linea me stesso, ma al contrario per pagare sia pure inadeguatamente e in ritardo un debito di gratitudine, desidero qui ricollegare l'egregia curatrice al suo maestro Ezio Raimondi, che non per niente firma la Premessa alla raccolta di scritti jollesiani. Ben prima che ne uscisse la traduzione italiana (1980), Raimondi aveva spronato chi scrive alla lettura di Einfache Formen, nonché della Einleitung al Decameron tradotto da Wesselski (Leipzig, Insel, I92I), infine dei Literarische Travestien. Tanto mi premeva rammentare, per dare a un maestro di statura incontestabile e rara generosità intellettuale come Raimondi quanto gli è dovuto, credo non certo solo dal sottoscritto. Ora, tutti questi testi (e altri, non meno decisivi per intendere il percorso intellettuale di Jolles), per l'attenta cura della Contarini, che firma anche una dettagliata e ricca Introduzione, sono di nuovo disponibili per il pubblico italiano, in versione sempre più affidabile di quanto non fosse finora. Segnalo a questo proposito, per menzionare solo i due casi forse più significativi, che la su citata introduzione all'edizione tedesca del Decameron è qui pubblicata ${ }^{4}$ secondo la successiva (I923) versione olandese rivista dall'autore con l'apporto di Huizinga; e che Forme semplici viene proposto in una nuova versione, fedele alla terza edizione (1958).

Si suole frequentemente avvicinare l'esperienza di Jolles al formalismo e alle sue più recenti propaggini, in primo luogo lo strutturalismo, ma si tratta di un accostamento indebito, per almeno un doppio e importante ordine di motivi: innanzi tutto perché la morfologia di Jolles, se la si vuole definire tale, è un'analisi storica delle "forme», sottratta perciò stesso alle sirene della sincronia, e anzi calata costantemente nel tumulto della storia, senza poi considerare il fatto, caratteristico del personaggio, che il passaggio dalle "forme semplici» alle "forme artistiche» è già di per sé un transito che esige la riflessione sulla diacronia, essendo per di più le stesse «forme semplici» lette da Jolles non come monadi incomunicabili, ma come insiemi in movimento verso contatti con esperienze limitrofe.

3. Per tutte queste informazioni, oltre alla Cronologia in coda al volume (ivi, pp. 453-457), cfr. A. Bodar, Labirinto europeo. Per una biografia critica di A. Jolles, «Intersezioni», VIII, 1988, pp. I55-I70, nonché B. Emmrich, "voce” Jolles, André (in tedesco), «Sächsische Biografie», edizione online (http://www.isgv.de/saebi/) [ultimo accesso: 9.6.2016].

4. A. Jolles, I travestimenti della letteratura, cit., pp. 57-II6. 
Significativo il fatto che, già anticipando il principio di "forma semplice», Jolles scriva: "Alcune di queste forme, come il proverbio, vivono quasi un'esistenza solitaria, da eremiti. Altre, al contrario, mostrano una singolare propensione all'unione e alla fusione con altre forme simili. Da questa tendenza prendono corpo formazioni più ampie, colonie o organismi che

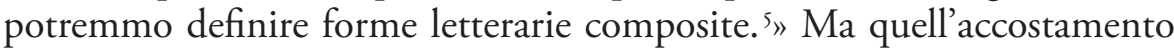
col formalismo è fallace anche perché primario è in Jolles, piuttosto che semplicemente il testo, la risposta di quest'ultimo alle esigenze psichiche, sociali, intellettuali, tanto del soggetto che dell'assetto sociale. Non solo dunque quella di Jolles è una morfologia storica, ma essa può venire ulteriormente circoscritta rilevando il fatto che è antropologicamente, piuttosto che solo testualmente, connotata. Beninteso, tale disposizione può anche venire interpretata come uno dei punti di debolezza della ricostruzione di Jolles, ciò che spiegherebbe, dopo il clamore della prima edizione italiana di Einfache Formen, il silenzio che ha troppo sbrigativamente avvolto, almeno nel nostro Paese, quella proposta.

Si deve certo dire che la parabola intellettuale di Jolles rimane sostanzialmente fedele a se stessa. Si leggano alcune righe di Folklore e scienza dell arte, frutto di uno Jolles solo venticinquenne (I897):

Il nucleo della mia ricerca consisteva in particolare nel mettere a confronto i fattori artistici presenti in forma embrionale all'inizio di una cultura, che non hanno ancora la forza di svilupparsi come arte, e l'arte al suo più alto grado di sviluppo. Mi chiedevo inoltre da quali fattori culturali fosse anticamente dipendente l'arte che a uno stadio successivo appariva ormai interamente distaccata dalla cultura ${ }^{6}$.

Le domande che qui si pone Jolles non sono almeno in parte le stesse cui cercherà in seguito di rispondere il saggista di Forme semplici? A quel libro Jolles arriverà con una serie di aggiustamenti progressivi di tiro, sui quali principalmente, potendosi dare per scontata la conoscenza dell'opera più nota, mi soffermerò in questa sede ${ }^{7}$. I testi cui farò riferimento sono pertanto il già citato $I l$ Decameron di Boccaccio, e La fiaba nella letteratura occidentale moderna ${ }^{8}$, entrambi, fino all'edizione della Contarini, inediti in veste italiana? .

5. Ivi, p. 59 .

6. Ivi, p. 5 .

7. Su questo cfr. S. Contarini, "Forme artistiche»: Jolles e la teoria della novella, in Boccaccio e i suoi lettori. Una lunga ricezione, G. M. Anselmi et al. (a cura di), Bologna, Il Mulino, 2013, pp. I09-I27.

8. A. Jolles, I travestimenti della letteratura, cit., pp. II7-2I9.

9. A parte il primo, di cui si è detto, il secondo esce a puntate in «De Gids» tra il 1922 e l'anno seguente. 
Il saggio sul Decameron necessita di un'avvertenza preliminare: esso è stato inizialmente concepito come presentazione, per il pubblico germanofono, del capolavoro di Boccaccio, un testo col quale, generalmente, quello stesso pubblico ha una confidenza senz'altro elevata, ma non paragonabile a quanto accade con la Commedia, e forse anche col Canzoniere, se si tiene presente la robusta tradizione del petrarchismo tedesco (che peraltro non al solo Petrarca lirico si rivolge; lo stesso dovrebbe dirsi del Boccaccio latino, per ovvî motivi nei secoli della prima modernità più familiare alla cultura tedesca dell'autore volgare $)^{\mathrm{IO}}$. Il dislivello evidentemente ha a che fare anche cogli squilibrî d'informazione caratterizzanti quei tempi, non solo nel mondo tedesco, ma nel nostro stesso Paese. Poiché la consapevolezza della sofisticata operazione intellettuale del Decameron è fatto recente, non deve stupire che Jolles si senta in dovere di prendere le mosse da informazioni sulla biografia di Boccaccio, e lo faccia cogli insufficienti e ingannevoli mezzi a quei tempi disponibili, basandosi (ma cautamente) su quanto Boccaccio dice di sé nelle proprie opere. Ma gli inevitabili scompensi legati a una situazione di oggettiva arretratezza dell'informazione sono ampiamente risarciti da affondi tuttora degni di massima attenzione. Mi soffermo solo su alcuni di essi.

Jolles sorprende il lettore per la curvatura del tutto particolare cui egli sottopone la propria interpretazione del Decameron. Chi è abituato a leggerlo in veste di storico delle forme qui rimane vantaggiosamente spiazzato. Certo, quell'attenzione neppure in questo caso si allenta (si vedano le pagine tuttora memorabili sulle diverse tipologie dei racconti a cornice, nelle quali si ritrova il volto dello studioso che è più familiare al lettore di Forme semplici); né Jolles, forte della appassionata discussione tedesca sulla novella, da Goethe, agli Schlegel, a Tieck, rinuncia a una sua efficace definizione del genere («Per novella intendiamo la rappresentazione di un fatto o di un avvenimento di significato chiaro e intenso, che suscita nel lettore un'impressione di verità. Tale avvenimento viene tradotto dalla novella in una forma che privilegia la rappresentazione dell'evento sui personaggi

Io. Per la fortuna tedesca di Boccaccio, al sempre utile W. Hirdt, Boccaccio in Germania, in F. Mazzoni (a cura di), Il Boccaccio nelle culture e letterature nazionali, Firenze, Olschki, 1978, pp. 27-51, si può ora affiancare (ma non ho potuto consultarlo direttamente) L. Rubini Messerli, Boccaccio Deutsch. Die DekameronRezeption in der deutschen Literatur (I5.-I7. Jahrhundert). Bd. I: Untersuchung; Bd. 2: Texteditionen, Katalog der handschriftlichen und gedruckten Überlieferung, Bibliographien, Register und Verzeichnisse, Amsterdam, New York, Rodopi, 20I2. Cfr. inoltre Ch. Bertelsmeier-Kierst, Zur Rezeption des lateinischen und volkssprachlichen Boccaccio im deutschen Frühhumanismus, e N. Henkel, Boccaccio, "Decamerone» IV I in der lateinischen Verfassung des Filippo Beroaldo. Mit einer Edition des Texts, in G. Boccaccio in Europa. Studien zu seiner Rezeption in Spätmittelalter und Früher Neuzeit, A. Aurnhammer, R. Stillers (Hg.), Wiesbaden, Wolfenbüttel, Harrassowitz, Herzog August Bibliothek, 20I4, rispett. pp. I3I-I53 e I55-I8I. 
coinvolti») ${ }^{\mathrm{II}}$. Ma, complice la necessità di rendere edotto il lettore su un libro più celebre (negativamente) che letto (ne viene fatto cenno proprio in apertura dello scritto), le mire di Jolles sono qui ben diverse. Egli vuole innanzi tutto fare piazza pulita di una lettura del Decameron come di un libro di mero intrattenimento, e insiste a più riprese sul fatto che la raccolta è invece caratterizzata da una forte tensione morale (s'intende, sempre rigorosamente implicita), tanto da poter concludere:

[...] nel testo di Boccaccio due antiche forme della poesia, la cornice e il racconto a catena confluiscono in una sola, che rivela le sue profonde radici nella cultura popolare, e io non sono venuto meno alla promessa iniziale di dimostrare che anche il Decameron è un libro morale ${ }^{12}$.

Chi oggi si confronti col Decameron può solo avere una riserva sulla cultura popolare come fatto attivamente operante, atteso il fatto che i rilevamenti effettuati sulle stratificate intertestualità boccacciane hanno portato alla luce, e tuttora fanno emergere, le basi classicistiche della incessante riscrittura del capolavoro. Ma il resto del discorso è, invece, assai avanti sui tempi, se si pensa che tuttora fatica a farsi strada l'idea di un libro che abbia traguardi diversi dal disimpegno. Ciò perché ci si rifiuta di fare i conti con un progetto che, malgrado la venerazione di Boccaccio per Dante e Petrarca (una venerazione che lo ha portato a tentare di conciliare l'inconciliabile), è di fatto radicalmente diverso da quello degli altri due grandi. Finché non ci si sarà liberati dalla pigra e mistificante idea di un Boccaccio passivo imitatore di Dante e pallido satellite di Petrarca (il che non significa, evidentemente, non fare i conti fino in fondo su quei rapporti, appunto più agonistici di quanto spesso si supponga), non se ne comprenderà la effettiva statura ${ }^{13}$. Se si vuole percepire quanto Jolles fosse in anticipo sui tempi in relazione all'impegno morale e intellettuale che presiede al Decameron, basta leggere attentamente quest'altro passo:

Quando alla fine i dieci decidono di separarsi, Panfilo può parlare a ragione di una relazione tra fratelli e sorelle; sebbene siano state narrate storie contrastanti, nonostante abbiano bevuto e mangiato insieme, cantato e giocato, attività che possono spingere il debole alla licenza, né tra i giovani né tra le fanciulle è mai accaduto alcunché

II. A. Jolles, I travestimenti della letteratura, cit., p. I03. Una rassegna delle prese di posizione tedesche del primo Ottocento sul genere novellistico in E. M. Meletinskij, Introduzione alla poetica storica dell'epos e del romanzo, Bologna, Il Mulino, 1993, pp. 255-318 (part. pp. 255-263).

I2. A. Jolles, I travestimenti della letteratura, cit., p. II4.

13. Affronto la questione in R. Bragantini, Petrarch, Boccaccio, and the Space of Vernacular Literature, in corso di stampa in Petrarch and Boccaccio. The Unity of Knowledge in the Pre-Modern World, I. Candido (a cura di), Berlin, De Gruyter Mouton. 
di biasimevole. Sì, Boccaccio sa molto bene che le cose che racconta assumono in letteratura un significato diverso che nella vita ${ }^{14}$.

Jolles riepiloga qui fedelmente il discorso di Panfilo (X Concl., 2-7); importante è innanzi tutto il fatto che la proposta di sciogliere la compagnia, formulata da quel narratore, intenda evitare il rischio che al selezionato gruppo si associno individui che non ne rispettino le leggi di decoro ed eccellenza intellettuale, così confermando la radice stoica ed esclusiva (prevalentemente senecana) della sodalitas decameroniana (fa testo la novella di Guido Cavalcanti, oggetto di uno specifico studio incluso nel volume qui discusso) ${ }^{\mathrm{I}}$. $\mathrm{Ma}$ anche va aggiunto che l'accettazione, da parte della compagnia dei narratori, della decisione del re dell'ultima giornata, rimarca quanto di «utile» e di «onesto» (X Concl., 8) è in quel consiglio; utilità e onestà sono non solo esplicitamente invocate o chiaramente implicate nel discorso iniziale di Pampinea (I Intr., 53-72), ma ancora richiamate nella Conclusione dell' Autore, ${ }^{1} 4^{16}$. Le affermazioni di Jolles risultano dunque, stavolta sul terreno della piena fedeltà testuale, perfettamente condivisibili (mentre invecchiata e non più sostenibile, benché allora moneta corrente e per lungo tempo ancora valida, pare la lettura del Decameron come organismo testuale governato da una tensione ascensionale paragonabile a quella della Commedia). Il punto più rilevante del passo su selezionato è tuttavia un altro: quando Jolles, nell'ultimo segmento di quello stesso passo, avverte acutamente la separatezza, in Boccaccio, di esperienza letteraria ed esistenza, dimostra di aver inteso alla perfezione la specifica natura della morale che presiede al libro, e di tenersi alla larga dalla trappola che il sottotitolo d'autore (il Decameron, nell'autografo berlinese, è "cognominato prencipe Galeotto» tanto all'inizio che alla conclusione della raccolta) vorrebbe tendergli. I dieci narratori non hanno commesso l'errore mimetico di Francesca e Paolo, poiché hanno accuratamente evitato di sovrapporre, indebitamente, letteratura e vita ${ }^{17}$.

Di rara intelligenza è anche l'affermazione, più volte espressa, anche se non compiutamente articolata, della preminenza dell'amicizia sull'amore

I4. A. Jolles, I travestimenti della letteratura, cit., pp. II2-II3.

15. Ivi, pp. 44-56. Sulla vicenda del concetto di sodalitas nell'antichità cfr. L. Pizzolato, L'idea di amicizia nel mondo antico, classico e cristiano, Torino, Einaudi, I993. Quanto alla presenza di Seneca, cfr. G. Velli, Seneca nel "Decameron", "Giornale storico della letteratura italiana», I68, I99I, pp. 32I-343; Id., Memoria, in Lessico critico decameroniano, R. Bragantini, P. M. Forni (a cura di), Torino, Bollati Boringhieri, 1995, pp. 222-248 (saggio che allarga lo sguardo, anche oltre Seneca, alla riscrittura boccacciana della classicità latina).

I6. Su questi punti cfr. P. Cherchi, L'onestade e lonesto raccontare del "Decameron», Fiesole, Cadmo, 2004.

17. Cfr. L. Battaglia Ricci, Ragionare nel giardino. Boccaccio e i cicli pittorici del "Trionfo della morte», Roma, Salerno, 1987, part. pp. I79-198. 
tanto nella vita che nel capolavoro narrativo di Boccaccio («[...] nella vita di Boccaccio l'amicizia ha avuto un ruolo più grande dell'amore»; "[...] Amore è una delle grandi divinità del libro, ma non la maggiore» ${ }^{18}$ ). Per quanto possa parere strano, se non addirittura incomprensibile, la semplice verità di tali proposizioni stenta ancora a trovare adeguato posto nella odierna lettura del Decameron; di essa solo mi preoccupo, dando per scontata la conoscenza del ruolo avuto dall'amicizia nella biografia di Boccaccio (caso mai sorprendendomi che, pur tenendosi lontani da inerti trasposizioni dalla vita dell'autore al suo testo, non si sia compiuto il passo seguente). Eppure il libro, lo attesta a chiare lettere il Proemio, nasce come disobbligo nei confronti degli amici che hanno alleviato la sofferenza amorosa di chi lo scrive (ulteriormente precisando quanto appena detto, qui non importa affatto che ci si trovi di fronte a ricostruzioni fittizie della vicenda amorosa di quest'ultimo; importa invece l'impegno solenne della restituzione del beneficio amicale) ${ }^{19}$. Jolles non insiste su questo punto, ma un passo, giusto alla fine del suo lungo saggio, mostra che ha tenuto fede a quanto quelle sparse affermazioni suggeriscono:

Gli esempi di amicizia e di liberalità, ma soprattutto della più rigorosa autodisciplina e del più nobile autocontrollo, si pongono in stridente contrasto con l'inizio dissoluto e privo di saggezza. Il vittorioso Carlo d'Angiò che con l'ausilio di un buon consigliere non soggiace a un capriccio, anche nei confronti di un avversario politico, e Pietro d'Aragona, che non si prende gioco di una giovane innamorata di lui, ma la guarisce con un bacio in fronte della sua follia amorosa, non sono forse eroi nel senso più alto, non ci offrono forse le ragioni per lasciarci alle spalle sorridendo le novelle precedenti? ${ }^{20}$

Stranamente, Jolles non fa qui menzione della novella di Tito e Gisippo (X 8), che offre il più fulgido riscontro di quanto da lui asserito. Accomunata a quelle indicate nel passo trascelto, e letta come correzione magnanima della tragica opposizione tra amicizia e amore nella novella di Guglielmo Rossiglione e Guglielmo Guardastagno (IV 9), quella novella sancisce, insieme a molte altre dell'ultima giornata, la supremazia dell'amicizia sull'amore, e sugli stessi legami familiari, come testimonia con reiterata forza il suo sigillo (X 8 III-II9).

Infine, merita un breve indugio la sezione del saggio dedicata alle fonti. Rilevando l'utilità, e al tempo stesso rimarcando le debolezze, del vecchio

I8. A. Jolles, I travestimenti della letteratura, cit., rispett. pp. 88 e II3.

19. Cfr. R. Bragantini, Ancora su fonti e intertesti del "Decameron»: conferme e nuovi sondaggi, in corso di stampa negli Atti del Convegno internazionale su Boccaccio: gli antichi e i moderni, Milano, Università Statale, 9-IO maggio 2016.

20. A. Jolles, I travestimenti della letteratura, cit., p. II5. 
libro di Marcus Landau (Die Quellen des Dekameron, I884²), Jolles, che, interessato com'è alle forme in perpetuo divenire, da quel tipo di ricerche è lontano anni luce, mette a fuoco il problema nella giusta prospettiva, anche se con un frasario che risente inevitabilmente del tempo:

per comprendere l'operazione del Boccaccio nella giusta maniera dobbiamo assumere il termine «fonte» in un senso più ampio. Non si tratta più anzitutto di stabilire da dove abbia tratto, o avrebbe potuto derivare il singolo racconto, ma di cominciare a indagare i suoi antecedenti spirituali, gli ambiti culturali in cui è cresciuta, nel tempo, la figura del narratore ${ }^{21}$.

In effetti, se sostituiamo ad «antecedenti spirituali» i concreti autori i cui testi Boccaccio ha spesso copiato di suo pugno, o comunque sicuramente consultato o postillato, e ad «ambiti culturali» la conoscenza, che oggi abbiamo incomparabilmente più approfondita, del suo reticolo di amicizie e contatti intellettuali, la questione è impostata nel modo più corretto $^{22}$. Per affrontare il punto, suggerisce Jolles, occorre smettere di pensare a un autore che preleva in modo inerziale segmenti narrativi da testi altrui, e avere invece in mente, come per l'appunto lo studio degli autori introiettati da Boccaccio obbliga a fare, un processo in cui la memoria culta è inseparabile dall'inventio del narratore. Chi, con superciliosa condiscendenza, fa vista di credere che ciò significhi apporre un cartellino segnaletico a ogni lacerto narrativo decameroniano, da riporre poi nel magazzino delle merci dismesse, vuole attribuire ad altri l'uso di strumenti impolverati che sono invece tuttora i suoi, e difficilmente può accostarsi alla natura parodistica (nel senso più strettamente etimologico del termine) del libro e dei suoi alambicchi intertestuali, tanto meno produrre un commento adeguato alla sfida lanciata dalla raccolta. Detto in altri termini: se per la Commedia e il Canzoniere l'individuazione degli intertesti è attitudine corrente, tanto più nei commenti odierni, per quale ragione non si deve effettuare la medesima operazione per un testo come il Decameron che, sia detto qui di passata, oltre ad avere una tradizione esegetica purtroppo non comparabile con quei due monumenti, pone difficoltà non inferiori a essi, e precisamente per il fatto che, malgrado la conoscenza della prassi scrittoria e dell'enciclopedia mentale di Boccaccio

2I. Ivi, p. 79 .

22. Mi limito a rimandare, per questi fatti, ad A. Mazza, L'inventario della "parva libraria" di Santo Spirito e la biblioteca del Boccaccio, "Italia medioevale e umanistica», 9, 1966, pp. I-74; a M. Signorini, Considerazioni sulla biblioteca di G. Boccaccio, "Studi sul Boccaccio", 39, 20II, pp. 367-395; infine, a M. Cursi, M. Fiorilla, G. Boccaccio, in Autografi dei letterati italiani. Le Origini e il Trecento, t. I, G. Brunetti, M. Fiorilla, M. Petoletti (a cura di), Roma, Salerno, 20I3, pp. 43-I03; nonché, per i rapporti intellettuali, a V. Branca, G. Boccaccio. Profilo biografico, Firenze, Sansoni, I977. 
si sia notevolmente incrementata negli ultimi tempi, ci si rifiuta di fare i conti con tali realtà?

La fiaba nella letteratura occidentale moderna, che la Contarini ha reso finalmente disponibile dopo la prima uscita in rivista, è chiaramente, come la curatrice indica, un cartone preparatorio a Forme semplici, benché l'ampio saggio ${ }^{23}$, che riguarda le principali letterature europee (nell'ordine di trattazione, che è di fatto anche il cronologico: l'Italia, la Francia, la Germania, mentre più in ombra, ma recuperate tramite frequenti agganci alle predette, stanno la Spagna e l'Inghilterra), si preoccupi di fornire innanzi tutto le coordinate per orientarsi in un terreno che proprio tra ultimo Ottocento e primo ventennio del Novecento la comparatistica viene esplorando e risistemando da una situazione estremamente confusa, mentre un nuovo filone, che si riversa nelle principali letterature occidentali con moto inarrestabile, è costituito dall'impresa di Antoine Galland e della sua versione delle Mille e una notte. L'assenza della raccolta dei Grimm è coerente col fatto che Jolles si concentra sulla fiaba d'autore, e infatti i raccoglitori dei Kinder-und Hausmärchen sono presenza ricorrente nelle Forme semplici. Peraltro non solo il saggio su cui mi soffermo è trampolino per il libro più noto, se si legge il rivelatore carteggio con Huizinga (I925). Nell'analizzare i possibili diversi modi di presentazione di un fatto pertinente alla quotidianità, Jolles sciorina con chiarezza le varie angolature da cui esso può venire traguardato; in tal modo ci si parano davanti le variabili del rapporto (termine da intendersi in senso tecnico-burocratico), della novella, del caso, del memorabile ${ }^{24}$, sul cui legame genealogico con le successive Forme semplici non è necessario insistere, dato che alle due ultime designazioni sono dedicate pagine specifiche di quel libro, da cui la novella, che è per Jolles "forma artistica», è esclusa. Ma che tale attenzione sia pienamente allertata già nel saggio sulla fiaba mostra chiaramente un passaggio che si legge proprio in apertura dello scritto:

nella forma e nel contenuto contiene [scil.: la novella di Boccaccio] un certo numero di elementi che la uniscono alla letteratura antica, al suo interno si celano residui di precedenti conglomerati letterari medievali come i Gesta Romanorum o i Sette savi, racchiude frammenti di aneddoti morali che venivano raccolti per essere usati nelle prediche o nei libri edificanti come exempla o illustrazioni, residui dell'epica francese che conosciamo attraverso i fabliaux, delle vite dei santi e altro ancora, ma nondimeno è un fenomeno in sé concluso, che possiede uno sviluppo indipendente e un'esistenza autonoma. Lo avvertiamo subito se paragoniamo il Decameron con le

23. A. Jolles, I travestimenti della letteratura, cit., pp. II7-2I9.

24. Cfr. ivi, pp. 238-240. 
raccolte precedenti, le Cento novelle antiche: la grande arte individuale di Boccaccio ha infuso nuova vita alla novella ${ }^{25}$.

Così è in effetti, e tanto più entro la cornice della morfologia storica di Jolles. Se si leggono in parallelo, per fare due soli esempî, la novella della donna di Guascogna e del re di Cipro (I 9), e quella di Melchisedech e del Saladino (I 3), e le si sovrappongono alle precedenti narrazioni del Novellino (rispettivamente LI e LXXIII), che Jolles cita secondo il titolo invalso dalla princeps (I525) curata da Carlo Gualteruzzi, ciò che salta subito agli occhi è non solo la quantità di appigli rappresentativi che, nel Decameron, ancorano il fatto a una specifica realtà storica, di contro agli irrelati sprazzi del Novellino, confezionati sotto vuoto spinto; ma anche (mi attengo, per brevità, alla sola seconda narrazione boccacciana) che la trattazione letteraria della vicenda incrementi in modo sbalorditivo tutti gli antecedenti (compresi quelli che sono di base allo stesso Novellino), con la registrazione finale della duratura amicizia tra il Saladino e Melchisedech, particolare decisivo e del tutto assente dalle molte versioni dell'episodio (ciò che incorpora la discussione sull'amicizia tra disuguali, così come essa è trattata nell'Etica Nicomachea, VIII 7 II58b-II59a, che Boccaccio conosce tramite la tomistica Sententia libri Ethicorum, da lui stesso copiata nel ms. Milano, Biblioteca Ambrosiana, A 204 inf., databile a un decennio precedente il Decameron). Quanto tutto ciò si attagli alla definizione jollesiana della novella su riportata non è necessario rimarcare; neppure occorre sottolineare come la prospettiva fornita da Boccaccio alimenti di incursioni problematiche il nudo referto dei precedenti ${ }^{26}$.

Ma la fiaba (del cui ampio saggio jollesiano seleziono, per economia, solo alcuni punti) risponde ad altre esigenze, e i suoi infiniti labirinti rendono arduo, se non impossibile, il tentativo di rintracciarne precise genealogie testuali (che infatti la moderna comparatistica ha abbandonato, sin dagli studî di Veselovskij, a favore di motivazioni di ordine psichico, capaci di spiegare la coincidenza pressoché perfetta di narrazioni nate in culture mai

25. Ivi, p. II8.

26. Resta fondamentale in proposito H.-J. Neuschäfer, Boccaccio und der Beginn der Novelle. Strukturen der Kurzerzählung auf der Schwelle zwischen Mittelalter und Neuzeit, München, Fink, 1969, purtroppo ancora pressoché sconosciuto al lettore italiano, se si eccettuano alcune pagine dedicate a Decameron, IV 9, in Il racconto, M. Picone (a cura di), Bologna, Il Mulino, 1985, pp. 299-308. Una sintesi di quanto trattato da Neuschäfer in quel libro si può inoltre leggere in Id., Boccace et l'origine de la nouvelle. Le problème de la codification d'un genre médiéval, in M. Picone, G. Di Stefano, P. D. Stewart (a cura di), Formation, codification et rayonnement d'un genre médiéval. La nouvelle, actes du colloque international de Montréal (McGill University, I4-I6 octobre 1982), Montréal, Plato Academic Press, I983, pp. IO3-IIO. 
venute a contatto reciproco ${ }^{27}$; si aggiunga che destinato a un frustrante fallimento è anche lo sforzo di classificazione delle diverse tipologie di un genere per eccellenza segnato dalla reiterazione. Rimane inoltre il problema centrale del genere stesso, quello della morale che gli è, in un modo o nell'altro, anche se in forme sempre diverse, sottesa. In questo senso già Straparola, che Jolles situa all'inizio della fioritura della fiaba moderna, nella sua specifica veste magica, pone un duplice ordine di problemi: perché da un lato non tutti i racconti delle Piacevoli notti possono essere raggruppati sotto il cartiglio fiabesco, posto che un discreto numero di essi sono novelle nel senso classico del termine; dall'altro, la morale conclusiva è sostituita dal giuoco di società degli enigmi (tutti e due i punti sono afferrati con mano sicura da Jolles). Il dirompente successo anche europeo delle Piacevoli notti rimane in un certo senso inspiegabile, né avrebbe potuto trovare altro terreno fertile che nella Venezia di metà Cinquecento (le principes delle prime due parti sono del I550 e I553; un solo ventennio dopo, la stampa della raccolta, che va infatti presto incontro a vistose restrizioni o sconcianti modifiche, sarebbe stata impossibile). Nell'affrontare il problema del testo, Jolles sostiene prudentemente il connubio tra cultura popolare e umanesimo ${ }^{28}$; una formulazione che, non dico la migliore conoscenza di Straparola, che rimane sostanzialmente un prodigioso fantasma della paraletteratura, ma le incrementate conoscenze del dibattito intellettuale e della vita letteraria ed editoriale cinquecentesca, non permettono oggi di condividere appieno. Tali risvolti sono tuttavia secondarî nell'analisi dello studioso, che lavora avendo mire in buona parte diverse, coincidenti con lo sforzo di individuare in cosa consista la fiaba di magia, più specificamente ancora, il racconto di fate, di cui Straparola viene giustamente ritenuto il moderno antesignano (certo, esiste il precedente latino di Morlini, da cui Straparola attinge, direttamente traducendole, ben 23 delle sue fiabe ${ }^{29}$; ma tra l'una e l'altra esperienza, la storia della rispettiva fortuna italiana ed europea lo dimostra, il divario è enorme). Tale risultato è pienamente raggiunto, tramite la registrazione degli scarti che la fiaba di magia effettua nei confronti della pur sempre presente tradizione novellistica.

27. Cfr. A. N. Veselovskij, Poetica storica, prefazione di D’A. S. Avalle, trad. dal russo e note di C. Giustini, Roma, Edizioni e/o, I98I, pp. 276-29I; gli stessi saggi in A. N. Veselovskij et al., La cultura nella tradizione russa del XIX e XX secolo, D’A. S. Avalle (a cura di), Torino, Einaudi, 1982, pp. 69-87.

28. Nella sezione straparoliana del saggio Jolles segue dappresso, mutuandone anche alcune imprecisioni, la pionieristica tesi di Dottorato di F. W. J. Brakelmann, Giovan Francesco Straparola da Caravaggio, Göttingen, Universitäts-Buchdruckerei von E. A. Hut, 1867.

29. Cfr. ivi, p. 33. 
Nell'affrontare Basile e il Cunto de li cunti la guida di Jolles è naturalmente costituita, come da dichiarazione di Jolles stesso, dal Croce dei Saggi sulla letteratura italiana del Seicento ${ }^{30}$; per quanto riguarda il testo, è verosimile Jolles si rifaccia alla versione tedesca di Felix Liebrecht (I846) prefata da Jacob Grimm, non essendo ancora disponibile, all'epoca della pubblicazione del suo lavoro, la splendida versione crociana del I925, e dovendosi ritenere piuttosto improbabile che potesse consultare senza intoppi l'edizione, sempre crociana, del I89I, contenente il testo originale delle sole due prime giornate; a meno che l'accenno che Jolles fa alla difficoltà del dialetto napoletano di Cortese e Basile ${ }^{3 \mathrm{I}}$ non debba far propendere per una terza, e forse plausibile, ipotesi, una lettura del testo napoletano alleviata nelle sue impervietà dalla versione di Liebrecht ${ }^{32}$. Comunque stiano le cose, l'excursus su Basile non solo esibisce pagine suggestive e convincenti sul Cunto, ma anche, come ricordato, sondaggi in cui già si percepisce il caratteristico rovello formale che si dispiegherà pienamente nell'opera maggiore dello studioso. Il punto in cui Croce e Jolles divergono, almeno parzialmente, è costituito dal dilemma, che inevitabilmente viene a galla di fronte a simili prove, sulla naturalezza o artificiosità del dialetto napoletano di Basile, e, più in generale, sulla purezza popolare o contaminazione culta del suo esercizio fiabesco. Secondo Croce, Basile evita talora accuratamente l'uso di parole italiane di uso corrente nella Napoli primoseicentesca; l'osservazione è sostanzialmente condivisa da Jolles, che però rimarca, con forza ancora maggiore di quanto Croce non faccia, il volto eminentemente parodistico del Cunto. Le due maschere dell'autore seicentesco, il poeta cortigiano e Gian Alesio Abbattutis, non giuocano a rimpiattino l'una con l'altra, ma sono appunto un capolavoro di equilibrismo parodistico (della prima maschera verso la seconda, e viceversa). Scrive Jolles:

Queste tre caratteristiche della scrittura, vale a dire il purismo forzato, il gusto della collezione e il demone della parodia dominano lo stile del Pentamerone, conferendogli qualcosa di popolaresco e di ampolloso insieme, un'anima rustica e raffinata nel contempo; in breve, esse fanno di Basile e del suo umorismo una delle maschere più divertenti della letteratura, mostrando come sotto gli abiti del cortigiano si celasse

30. Cfr. B. Croce, Saggi sulla letteratura italiana del Seicento, Bari, Laterza, I9II, part. pp. 3-I03; Jolles vi accenna a p. I3I.

3I. A. Jolles, I travestimenti della letteratura, cit., p. 132

32. L'utilizzo, in ogni caso, della versione di Liebrecht è reso tanto più probabile dalla lode senza riserve tributatale da Croce, Saggi sulla letteratura italiana del Seicento, cit., pp. 85-90; anche quanto detto da Jolles della fortuna di Basile in Europa, a es., per quanto riguarda le riprese di Wieland (A. Jolles, I travestimenti della letteratura, cit., pp. 203-204), si appoggia verosimilmente alle indagini di Croce, Saggi sulla letteratura italiana del Seicento, cit., pp. 8I-84. 
uno straordinario monello di strada, contemporaneo di Jacques Callot e di Pieter van Laar alias il Bamboccio ${ }^{33}$.

Poco oltre, tuttavia, in misuratissima contrapposizione a Croce, Jolles si interroga su un altro nodo capitale, se sia veramente corretto ritenere incolmabile, come per l'appunto tende a credere Croce, il divario tra la fonte popolare originaria delle fiabe e la sua trascrizione da parte di Basile, e a sua volta si domanda: "Una volta spogliata [scil.: la lingua del Cunto] dei suoi orpelli e dei suoi panni variopinti, non affiora invece qua e là qualche frammento dei materiali originari? ${ }^{34 » ~ B a s i l e ~ c o s t i t u i s c e ~ p e r ~ J o l l e s, ~}$ in questa prospettiva, un terreno d'indagine privilegiato, giacché in ogni fiaba la ricorrente scalarità di morale esplicita, racconto, proverbio conclusivo, si presta a essere interpretata appunto come una delle "forme letterarie composite» ${ }^{35}$, una sorta di sovradeterminante formale collocata all'apice dell'accidentato percorso tra "forma semplice» e "forma artistica».

L'occhio esperto di Jolles non si fa sfuggire una simile occasione e, mettendo a fuoco le sfilze sinonimiche caratteristiche di Basile (su cui tanto Imbriani che Croce già avevano attirato l'attenzione), nonché le incastonature culte che crivellano il dettato del Cunto, si sofferma sulla possibilità di distinguere, in uno stesso racconto, addirittura in uno stesso passo, differenti stratificazioni formali, non tanto cronologicamente (ciò è possibile solo a grandi linee), quanto appunto morfologicamente determinabili. Scrive in proposito Jolles:

[...] quasi in ogni fiaba [...] si ritrova un passo che propone una lezione morale, nella maggior parte dei casi legata a un proverbio, ovvero la morale della quale il racconto successivo diviene l'illustrazione. Abbiamo visto che la morale ritorna ripetutamente nel Decameron, ma senza assumere lo statuto di regola. Da questo punto di vista Basile appare dunque assai più conseguente di Boccaccio... o forse dovremmo piuttosto dire che la «fiaba» è più conseguente della «novella», e che la morale rappresenta una vecchia regola del racconto, di cui la forma nuova della novella toscana creata da Boccaccio testimonia solo in alcuni punti la sopravvivenza [...]. Si mettano tra parentesi le metafore e si avrà l'incipit originario della fiaba che si ritrova in ogni racconto ${ }^{36}$.

Senza che i due personaggi ne siano reciprocamente consapevoli, la posizione di Jolles e quella di Veselovskij sono in questo senso, pur nella diversità delle premesse, singolarmente vicine, se si legge quanto quest'ultimo afferma a proposito degli schemi della fiaba: «Sarebbe interessante

33. A. Jolles, I travestimenti della letteratura, cit., p. I34; corsivi nel testo.

34. Ivi, p. 136 .

35. Ivi, p. 59.

36. Ivi, p. I39; corsivi e virgolette nel testo. 
elaborare una morfologia della fiaba e seguirne lo sviluppo dai più semplici momenti narrativi sino alla loro combinazione più complessa. Potremmo allora renderci conto che più antica è la fiaba, più elementare è il suo schema e che, al contrario, più una fiaba è nuova più lo schema si complica» ${ }^{37}$. Jolles avrebbe potuto replicare che lo sviluppo della morfologia storica non esclude dei consapevoli passi indietro, ma la sostanza resta affine, se si pensa che Veselovskij non ha in mente, nel passo citato, la «forma artistica» della fiaba, ma appunto quella che Jolles individuerà poi come "forma semplice» del genere, ancora priva delle incrostazioni artistiche depositatevi dai suoi esercenti. Anche in questo senso Basile costituisce un caso a sé; per chiarire in che senso occorre rifarsi a un passo in cui, discutendo della fiaba rococò francese, Jolles asserisce:

Nella sua forma breve e in sé conclusa, essa rappresenta anzitutto una reazione al romanzo. Tutta la storia della letteratura testimonia l'antica ostilità tra il racconto lungo e quello breve. A volte le due forme prendono il posto l'una dell'altra, a volte proseguono parallele a prezzo di qualche scaramuccia, altre ancora qualcuno tenta di riavvicinarle alla ricerca di un'armonia non priva di tensioni ${ }^{38}$.

Jolles, cui certo non sono ignote le pagine di Schlegel sulla natura «ritardante» del romanzo e sulla riflessione critica e ironica che gli è storicamente connessa, almeno da Cervantes e dal Goethe di Wilhelm Meister in poi, ha qui presente un aggrovigliato gomitolo formale che vede da una parte quelle prove eccelse, dall'altro il repertorio, a noi noto solo mediatamente, delle fabulae milesiae, nonché le Metamorfosi di Apuleio, non per caso incunabolo della stessa narrazione boccacciana ${ }^{39}$. Non può perciò non sapere che le leggi che presiedono ai due modelli, racconto breve e romanzo, anche in ciò sono antitetiche: il racconto è intensivo, economico, secluso (ma il caso di raccolte di racconti costituisce una non indifferente eccezione a quest'ultimo punto); il romanzo, nella sua forma moderna inaugurata appunto da Cervantes ( $m$ a si potrebbe anche risalire più indietro), è invece estensivo, digressivo, autochiosatorio. Basile contraddice alle leggi imperanti nel genere fiabesco da lui praticato, intasando tanto di filatesse sinonimiche quanto di indugi glossatorî una forma narrativa da sempre a tal punto concentrata esclusivamente sull'azione dell'eroe, da abbattere senza alcuna remora di inverosimiglianza qualsiasi ostacolo

37. Il passo in A. N. Veselovskij, Poetica storica, cit., p. 277, nonché in A. N. Veselovskij et al., La cultura nella tradizione russa, cit., pp. 70-7I.

38. A. Jolles, I travestimenti della letteratura, cit., p. I6I.

39. Su quest'ultimo punto, cfr. I. Candido, Boccaccio umanista. Studi su Boccaccio e Apuleio, Ravenna, Longo, 20I4, senz'altro il lavoro più completo e aggiornato in materia. 
che a essa si contrapponga. Anche in questo senso si rivela la genuina sostanza della scrittura barocca di Basile. In maniera in fondo non troppo dissimile da quanto accade nell'Adone del da lui venerato Marino, che consapevolmente disgrega l'ordinata sequenza dei meccanismi narrativi della tradizione cavalleresca ed epica sostituendovi un sistema multicentrico di corrispondenze, Basile inceppa a bella posta, a ogni piè sospinto, la macchina perfettamente oliata del racconto fiabesco, diretta a non altro che alla linearità, priva di coordinate spaziali e temporali, dell'azione, immettendovi indugi sussultorî ${ }^{40}$.

Non è possibile seguire Jolles nella sua intera ricostruzione dei percorsi della fiaba moderna. Ma, poiché nelle sue pagine il problema della morale storicamente connessa a quella forma breve è il punto di convergenza delle diverse pratiche di quel genere, sarà sufficiente richiamare la sua definizione di La Fontaine come l'enfant terrible del secolo di Luigi XIV. Quel cartiglio si attaglia perfettamente tanto alle Fables che, ancor più, ai Contes. Chissà se Jolles sapeva ( $\mathrm{ma}$ il suo silenzio in materia induce a pensare il contrario) che quello stesso enfant terrible, ammalatosi gravemente nel febbraio 1693 (morirà tuttavia 26 mesi dopo) e ricevuta l'estrema unzione, sconfessò apertamente i suoi Contes con le parole seguenti (riportate però dal sacerdote che gli aveva recato il sacramento):

Monsieur, j'ai prié messieurs de l'Académie française, dont j'ai l'honneur d'être un des membres, de se trouver ici par députés, pour être les témoins de l'action que je vais faire. Il est d'une notoriété qui n'est que trop publique, que j'ai eu le malheur de composer un livre de Contes, infâme. En le composant je n'ai pas cru que ce fût un ouvrage aussi pernicieux qu'il l'est. On m'a sur cela ouvert les yeux, et je conviens que c'est un livre abominable. Je suis très fâché de l'avoir écrit et publié. [...] Je voudrais que cet ouvrage ne fût jamais sorti de ma plume et qu'il fût en mon pouvoir de le supprimer entièrement. Je promets solennellement, en présence de mon Dieu, que je vais avoir l'honneur de recevoir, quoique indigne, que je ne contribuerai jamais à son débit ni à son impression ${ }^{41}$.

Quale che sia il quoziente di verosimiglianza attribuibile a queste parole (ma, all'atto di spogliare il suo corpo per deporlo sul letto di morte, si trovò La Fontaine cinto di cilicio), esse mostrerebbero quello che una

40. Per Marino il punto di riferimento è costituito da G. Pozzi, Preliminari a Marino (fusione di due lavori risalenti al I97I e al 1973), ora in Id., Alternatim, Milano, Adelphi, I996, pp. 205-256; cfr. anche, dello stesso, la Guida alla lettura, in G. B. Marino, L'Adone, G. Pozzi (a cura di), con dieci disegni di N. Poussin, nuova edizione ampliata, 2 t., Milano, Adelphi, I988, II, part. pp. 2I-44 (identici estremi di pagine nel secondo tomo della precedente edizione: G. B. Marino, L’Adone, G. Pozzi [a cura di], 2 voll., Milano, Mondadori, 1976).

4I. Il passo in La Fontaine, Euvres complètes, 2 voll. (I, Fables, Contes et Nouvelles, édition établie, présentée et annotée par J.-P. Collinet; II, Euvres diverses, édition établie et annotée par P. Clarac, Paris, Gallimard, rispett. I99I e I958), vol. I, pp. CLXV-CLXVI. 
lettura attenta di quell'autore comunque dimostra: che, con tutte le sue premesse sul desiderio di procurare semplice piacere ai suoi lettori, egli fosse invece del tutto consapevole del fondo pericolosamente oscuro, quando non addirittura tenebroso, che può celarsi sotto la superficie apparentemente piana del racconto e della fiaba. 